\title{
Brightness indention: A novel compression mechanism in the luminance-brightness mapping
}

\author{
GERARD SCHOUTEN and FRANS J. J. BLOMMAERT \\ Institute for Perception Research (IPO), Eindhoven, The Netherlands
}

\begin{abstract}
In a previous study, Schouten and Blommaert (1995) explicitly showed that brightness constancy implies a substantial compression in the luminance-brightness mapping. Here it is argued that additional compression mechanisms are required for scenes with large luminance ranges. To trace these, a series of experiments was conducted which involved expansion of the luminance range. We used a simple spatial configuration consisting of a disk on a contourless homogeneous surround (Ganzfeld). The contrast between the disk and Ganzfeld acted as the independent variable, while the size of the disk was parametrically varied. Sequential dichoptical brightness matches with a reference were carried out for both the disk and the surround. The results reveal a compression mechanism which we term brightness indention. This indention, which has not previously been reported in the literature, only occurs if the Ganzfeld is less luminous than the disk, and it takes the form of a brightness decrease of the immediate surroundings of the disk.
\end{abstract}

The compression termed brightness constancy implies that contrast information (i.e., quantities related to luminance ratios within a scene) is primarily transferred to the brightness scale (Forsyth, 1990; Walraven, EnrothCugell, Hood, MacLeod, \& Schnapf, 1990). Classical data (e.g., Bartleson \& Breneman, 1967; Jameson \& Hurvich, 1961), as well as our measurements (Schouten \& Blommaert, 1995), are in agreement with this idea. Moreover, it was shown that for images consisting of (small) disks on a Ganzfeld, the brightness of the Ganzfeld is scarcely affected by the contrast of the disks, at least to the extent of one log-unit luminance increment or decrement. In other words, the Ganzfeld luminance is, to a first-order approximation, anchored on the brightness scale.

The principle of brightness constancy is visualized once more in Figure 1. This figure is meant to show clearly that there is no a priori reason to assume that brightness constancy is the only compression mechanism in the luminance-brightness mapping. In particular, it is expected that other compression mechanisms emerge when the luminance range of a scene is increased. The search for additional compression mechanisms constitutes the main issue of the investigation described in this paper. With respect to this, it should be remarked that natural scenes can have considerable luminance ranges - three decades are not exceptional (McCann, 1988). This is caused by the ubiquitous presence of spatially nonuniform illumination, shadows, highlights, and light sources in the scene. An adventitious item of this investigation concerns the anchorage on the brightness scale. For small scene elements on a

Correspondence concerning this article should be addressed to G. Schouten, Philips CFT, Building SAQ-2, P.O. Box 218, $5600 \mathrm{MB}$ Eindhoven, The Netherlands (e-mail: schouten_g@cft.philips.nl). vast homogeneous surround, the brightness of the surround is apparently not influenced by the contrast of the scene elements. However, is this still true when the size of such a scene element is increased until it occupies a substantial part of the entire visual field? Before these questions can be answered, experimental data for scenes, to which luminance-range expansion and size variation are applied, have first to be gathered.

\section{METHOD}

The experimental situation was largely similar to that of our brightness-constancy study. For details, we refer to Schouten and Blommaert (1995). Here, we restrict ourselves to an enumeration of the essentials.

A special-purpose optical apparatus (see Figure 2) was employed to present a disk surrounded by a Ganzfeld to the viewer's left eye. The size of the disk and its contrast with the Ganzfeld were varied. The largest disk we used was a $17.7^{\circ}$ circular field of view corresponding to the hole in the hemisphere. Size variations were achieved by using locking caps with variable circular apertures. These covers, which have about the same reflecting properties as the inner-sphere surface, were fitted to the back of the sphere. Since retinal blur caused by these locking caps is unavoidable, ${ }^{1}$ very small disks $\left(<2^{\circ}\right)$ were created directly on the monitor. ${ }^{2}$ Expansion of the luminance range implies enlargement of the contrast between the disk and the Ganzfeld.This can be accomplished by means of the neut ral density filters $F_{1}$ and $F_{2}$. The luminance-contrast setting is limited.The upper bound of the luminance contrast between the disk and the Ganzfeld is related to the integrating property of the Ulbricht sphere; the lower bound is determined by the diffuse reflective component of filter $F_{2}$. As pointed out in Figure 2, annoying specular reflections were avoided by orienting filter $F_{2}$ in an oblique direction. This left-eye image is called the test stimulus.

The right-eye configuration forms the reference stimulus. It consisted again of a $9^{\circ}$ circular background field with a fixed luminance $L_{0, \text { ref }}$ in an otherwise dark surround. In the middle of this background field, a disk of $1.2^{\circ}$ with a variable luminance $L_{\text {ref }}$ was superimposed. 


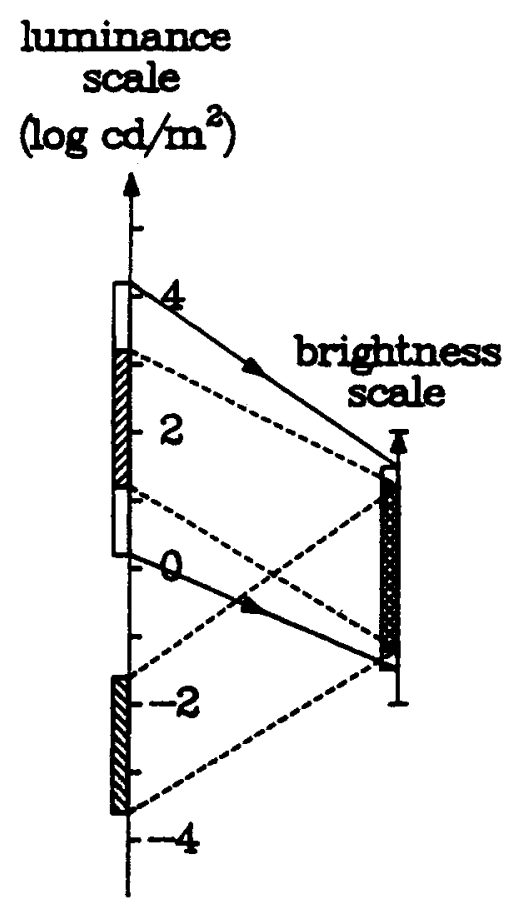

Figure 1. Illustration of perfect brightness constancy and the need for additional compression mechanisms. Attenuation of a luminance distribution with a constant factor results in a translation on a logarithmic axis.

In each of the following experiments, the observer's task was to adjust the luminance $L_{\text {ref }}$ of the reference disk until it was perceived as being equal to the brightness of a previously indicated field of the test stimulus. The specific brightness-match instruction ran as follows: "Make the brightness, i.e., the apparent lightdensity, of the reference disk and the relevant test element equal, disregarding as much as possible other areas in the visual field. Potential color and texture differences between the reference disk and the test element must be ignored." Note that since brightness increases monotonically with the luminance of the reference disk, the $L_{\text {ref }}$ scale is in topological correspondence with the brightness scale.

The left-eye test stimulus and the right-eye reference stimulus were presented sequentially. Most of the time, the observer viewed the test stimulus. Pressing a button caused the reference stimulus to appear for only $I \mathrm{sec}$, after which, the test stimulus reappeared. After each presentation of the reference stimulus, the observer reported whether the reference disk was darker or brighter than the specific test field. Next, while the observer again viewed the test stimulus, the experimenter adjusted the luminance $L_{\text {ref }}$ of the reference disk. This procedure was repeated until a match was obtained.

Two observers, F.B. and G.S., who also performed the brightnessconstancy measurements (see Schouten \& Blommaert, 1995), participated in these experiments. Both had uncorrected vision. At the time of the experiments, Observer F.B. was 43 years old and $\mathrm{Ob}$ server G.S. was 27.

\section{Test Stimuli and Procedure}

The test stimuli consisted of only two luminances - a disk with diameter $d$, surrounded by a Ganzfeld. As illustrated in Figure 3, two types of experiments were carried out. In the constant Ganzfeld (CG) experiments, the luminance of the disk, $L_{\mathrm{d}}$, was varied, while the luminance of the Ganzfeld, $L_{\mathrm{G}}$, was kept con- stant; in the constant disk (CD) experiments, $L_{\mathrm{G}}$ was varied, while $L_{\mathrm{d}}$ was kept constant. For the diameter $d$, values between $0.6^{\circ}$ and $17.7^{\circ}$ were chosen in three approximately equidistant steps on a logarithmic scale.

Although the Ganzfeld had a uniform luminance, it appeared markedly nonhomogeneous if the disk was more luminous than the Ganzfeld. In particular, the direct surroundings of the disk looked darker. In such cases, the observers were asked to perform brightness matches for three parts of the test stimulus: the far surround, the near surround, and the disk. For the near surround, both observers judged in effect the average brightness of an area equal in size to the reference disk and bordering directly on the test disk. The brightness of the far surround and the test disk gave a homogeneous impression. When the entire Ganzfeld was also perceived as being uniform, only two brightness matches were made-one for the disk, and one for the Ganzfeld.

For each experiment, four sessions were run, on at least two different days. During a session, which lasted about $45 \mathrm{~min}$, one brightness match was obtained for every contrast setting. We always started with the lowest setting of the variable luminance and proceeded with increasingly higher values. Within each session, the experimenter determined the order in which the different parts of the test stimulus were matched with the reference disk.

\section{RESULTS}

The results of the $C G$ and $C D$ experiments are shown in Figures 4 and 5, respectively. In both figures, $\log L_{\text {ref }}$ values are plotted as a function of the variable luminance $\left(L_{\mathrm{d}}\right.$ for the $\mathrm{CG}$ experiments, and $L_{\mathrm{G}}$ for the CD experiments). The value of the constant luminance (concerning either the Ganzfeld or the disk) is indicated by an arrow. The background luminance $L_{0 \text {, ref }}$ of the reference stimulus is denoted by a star on the ordinate. The symbols in all plots represent geometrical means of four $L_{\text {ref }}$ settings. Standard deviations of these means exceeding the symbol size are marked by vertical bars. Open circles are for brightness matches of the disk. Brightness matches of the far surround are denoted by squares with an upright cross $(+)$, and brightness matches of the near surround are denoted by squares with a diagonal cross $(x)$. In the case of a subjectively homogeneous Ganzfeld, the crosses are omitted.

The results in Figure 4 , for $d=0.6^{\circ}, 1.8^{\circ}$, and $5.2^{\circ}$, display similar tendencies. First, the brightness of the far surround is found to be more or less constant for variations of the luminance $L_{\mathrm{d}}$ over several decades. Second, the brightness of the disk increases monotonically with luminance $L_{d}$; the extent of this effect seems to diminish gradually with the size of the disk. Third, whenever the disk is more luminous than the Ganzfeld, the brightness of the near surround is locally suppressed. For $d=$ $17.7^{\circ}$, the brightness of the Ganzfeld gradually decreases as a function of the luminance $L_{\mathrm{d}}$.

The experimental results of Figure 5 indicate that the brightness of the Ganzfeld slowly increases as a function of its luminance $L_{\mathrm{G}}$. The brightness of the disk strongly declines with the Ganzfeld luminance. This effect is known as brightness induction (e.g., Heinemann, 1972; Horeman, 1965). Note that for $d=17.7^{\circ}$, on the one hand the brightness increase of the Ganzfeld is somewhat more pronounced, and on the other hand the 


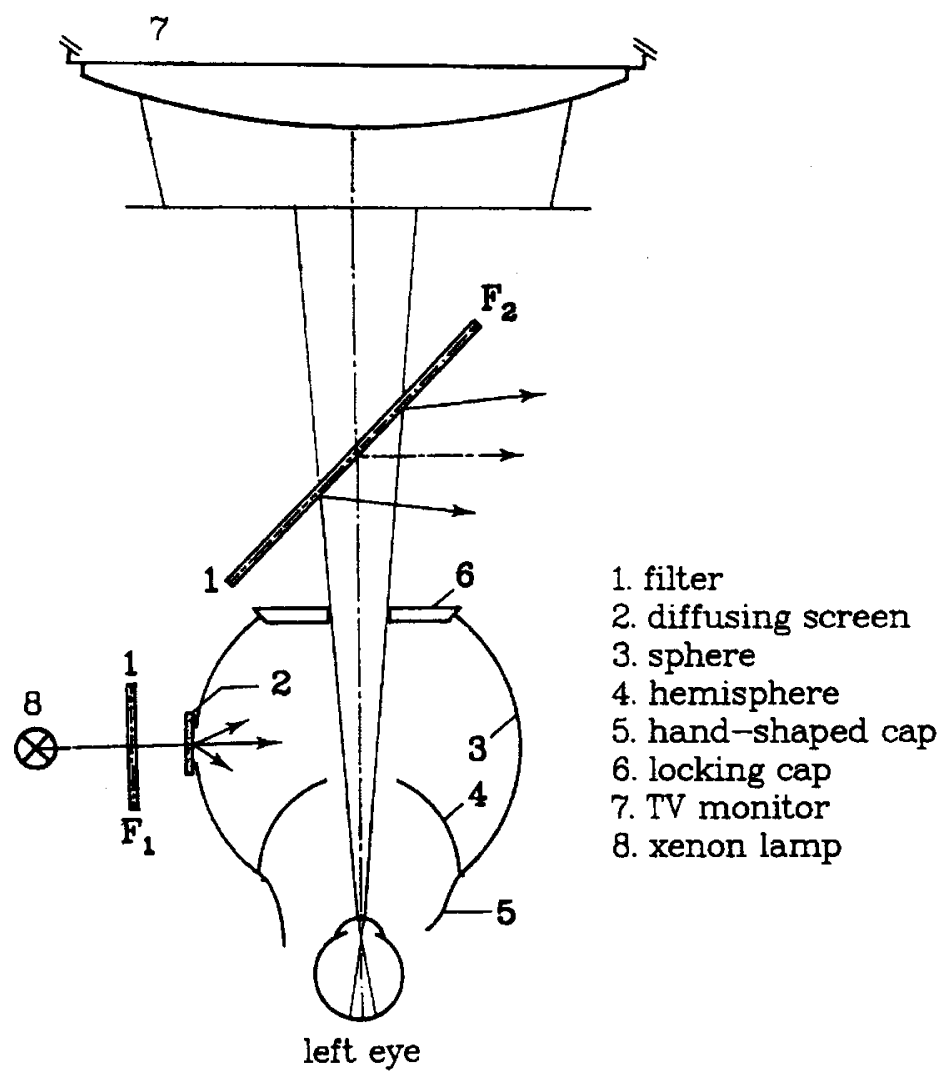

Figure 2. Simplified sketch of the optical apparatus (for the complete drawing, see Schouten \& Blommaert, 1995).

brightness decrease of the disk seems less dramatic. Again, as displayed in the graphs labeled $d=0.6^{\circ}$ and $d=1.8^{\circ}$, the visual system adopts a brightness indention around the disk if this field is more luminous than the Ganzfeld. Finally, it should be mentioned that there is an approximate mirror symmetry between the results of the $C G$ and $C D$ experiments.

\section{DISCUSSION}

\section{Brightness Indention}

During pilot measurements, both observers had the impression that if the luminance $L_{\mathrm{d}}$ of the disk was substantially higher than the Ganzfeld luminance $L_{\mathrm{G}}$, no single brightness value could be attributed to the surround. The phenomenon has an asymmetrical appearance; it only occurs if the Ganzfeld is less luminous than the disk, and leads to a brightness drop of the nearby surround. This lowering flattens out smoothly to the brightness level of the far surround. The strength of this brightness indention gradually increases with the contrast of the bright disk on the relatively dark surround. Furthermore, the spatial extent of the indention seems to scale with the size of the disk. As a rough estimate, the dimension of the indention equals the disk size. These observations probably explain why there is no visible bifurcation of the surround for $d=17.7^{\circ}$. At this point, it should also be emphasized that this ef- fect is quite distinct from the Mach-band phenomenon (e.g., Fiorentini, 1972). Two major differences are that (1) the appearance of Mach bands is symmetrical (i.e., both dark and bright stripes can occur), and (2) Mach bands are highly localized, with the width varying from a few minutes of arc to $10^{\prime}$ of arc at the most. Moreover, the appearance of Mach bands at the edges of an abrupt step change in luminance is debatable (e.g., Ratliff, 1984). ${ }^{3}$

If it is assumed that contrast information is unaffectedly transferred to the brightness scale, the effect of
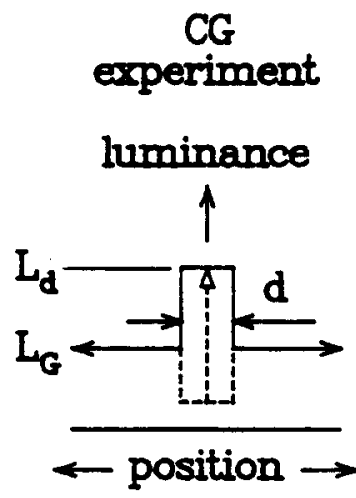
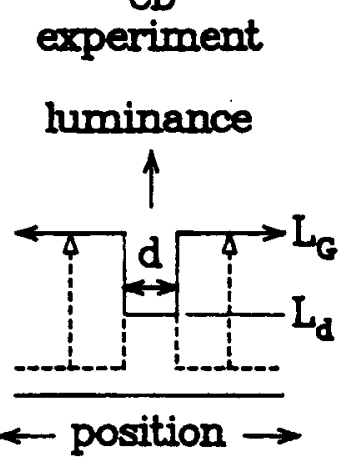

Figure 3. Luminance profiles of the test stimuli. The luminance variations applied in the constant Ganzfeld and constant disk experiments are indicated. 

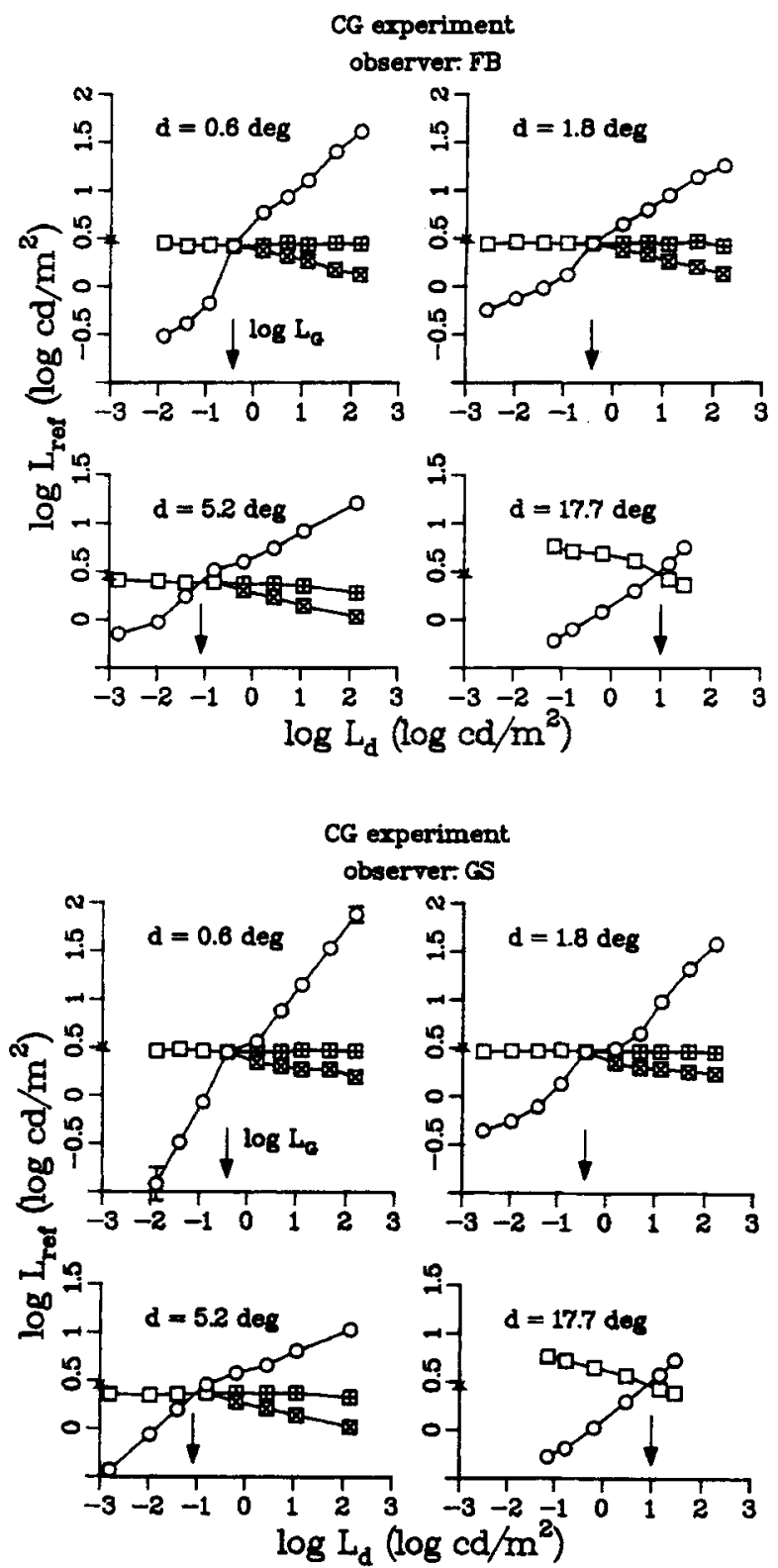

Figure 4. Results of the constant Ganzfeld experiments for $\mathrm{Ob}$ servers F.B. and G.S., with $\log L_{\text {ref }}$ values plotted as a function of $\log$ $L_{\mathrm{d}}$ for $d=0.6^{\circ}, 1.8^{\circ}, 5.2^{\circ}$, and $17.7^{\circ}$. Circles are for the disk, while the far surround is marked by a square with an upright cross, and the near surnound by a square with a diagonal cross. In the case of a subjectively homogeneous Ganzfeld, the crosses are omitted. The arrows indicate the constant values of $\log L_{\mathrm{G}}$. The background luminance of the reference stimulus is denoted by a star.

brightness indention is a reduction of the brightness range that is needed to represent the scene. Hence, it can be regarded as a compression mechanism in the luminancebrightness mapping. This is illustrated in Figure 6. Brightness indention implies that the concept of a oneto-one relationship (Fechner, 1860; Krueger, 1989; Stevens, 1957; Wagenaar, 1975) has to be ruled out for the luminance-brightness mapping. Moreover, the indention effect points to the necessity of incorporating spatial interactions into brightness models (see also McCann, 1988; Roufs, Blommaert, \& de Ridder, 1991).

This unexpected finding was discovered by systematically performing brightness matches for both the disk and the Ganzfeld. Other studies that used a single field on an
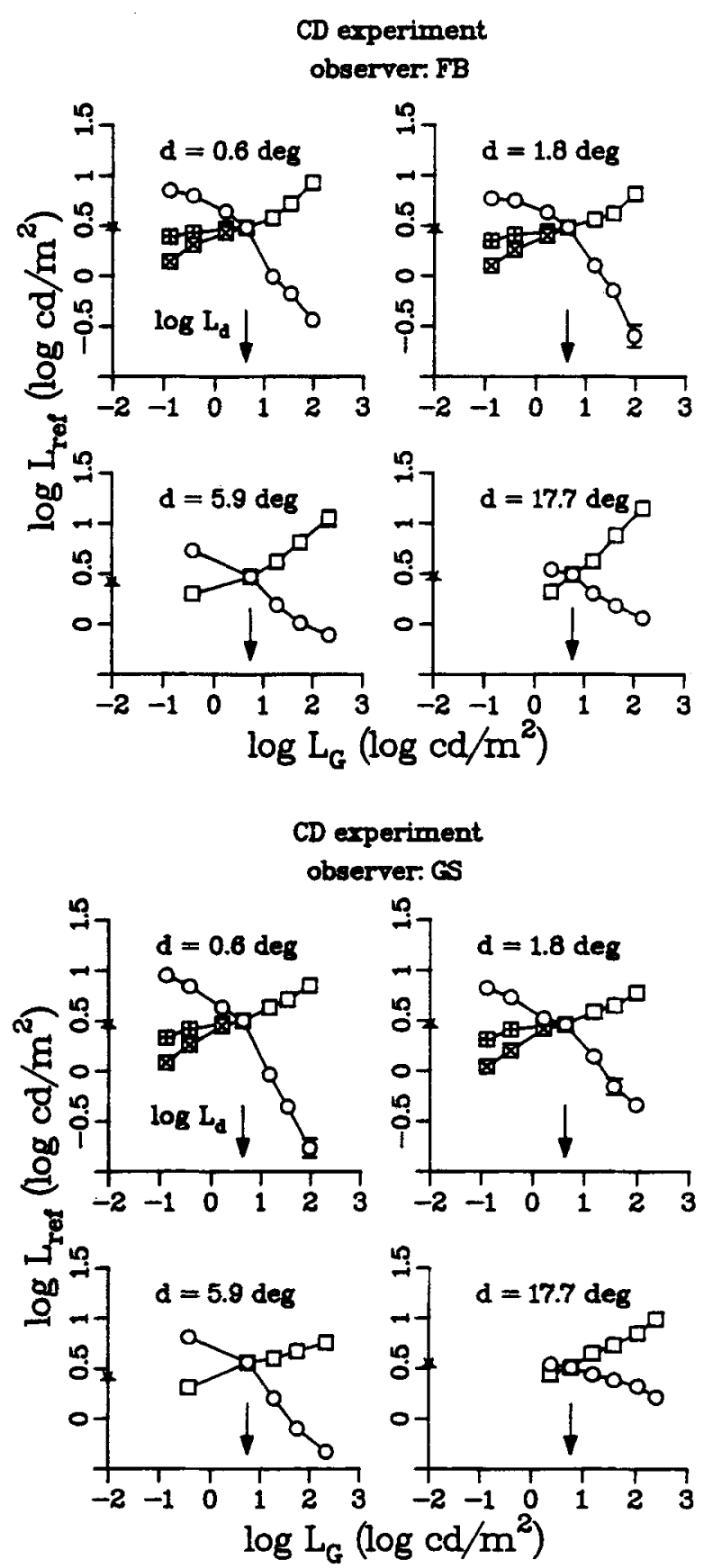

Figure 5. Results of the constant disk experiments for Observers F.B. and G.S., with $\log L_{\text {ref }}$ values plotted as a function of $\log L_{\mathrm{G}}$ for $d=0.6^{\circ}, 1.8^{\circ}, 5.9^{\circ}$, and $17.7^{\circ}$. Circles are for the disk, while the far surround is marked by a square with an upright cross, and the near surround by a square with a diagonal cross. In the case of a subjectively homogeneous Ganzfeld, the crosses are omitted. The arrows indicate the constant values of $\log L_{\mathrm{d}}$. The background luminance of the reference stimulus is denoted by a star. 


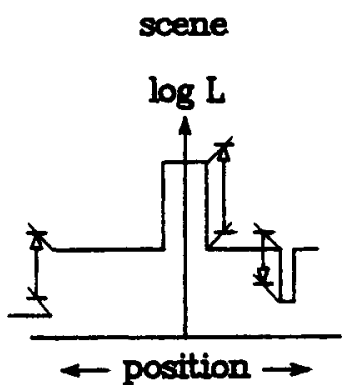

reconstruction I

reconstruction II
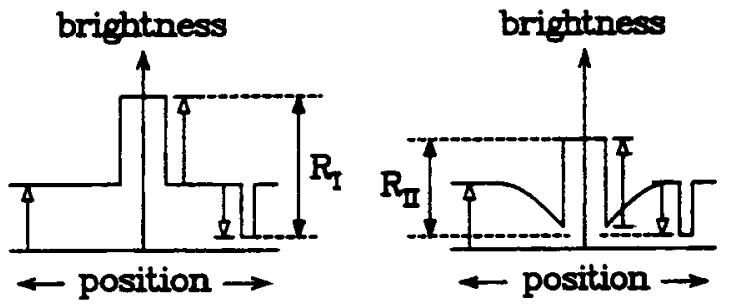

Figure 6. Illustration of the compression accomplished by brightness indention. Top: Luminance profile. The arrows symbolize contrast information extracted from the scene. The left-hand arrow represents a contrast with some reference level. Bottom: Brightness reconstructions assembled with the contrast information. In the reconstruction on the left, straightforward accumulation is applied; on the right, brightness indention is added. The occupied ranges in the sensoria are denoted by $R_{1}$ and $R_{1 \mathrm{II}}$, respectively.

extended uniform surround (e.g., Saunders, 1972) did not report the brightness-indention effect. This is probably due to the fact that only brightness measurements for that particular field were carried out. In order to quantify the brightness-indention phenomenon accurately, more precise measurements for the nearby surround are needed. Unlike our situation, this requires a small adjustable reference stimulus and the application of fixation marks in the test stimulus (Lowry \& DePalma, 1961).

\section{Internal Consistency}

The $C G$ and $C D$ experiments are closely related. Whereas a certain contrast setting for the CG experiments was achieved by adjusting the luminance $L_{\mathrm{d}}$ of the disk, this was done for the $C D$ experiments by changing the Ganzfeld luminance $L_{\mathrm{G}}$ in the opposite direction. The difference between the two types of experiments is to be found in the variation of the average luminance level. For small sizes of the disk, it can even be stated that the average luminance remains nearly constant in the case of CG experiments, and that it is almost inversely proportional to the luminance ratio $L_{\mathrm{d}} / L_{\mathrm{G}}$ for the $\mathrm{CD}$ experiments. If perfect brightness constancy holds (i.e., if there is invariance for changes of the illumination level), a pure mirror symmetry would be expected between the results of the $C G$ and $C D$ experiments, as plotted in Figures 4 and 5. This is not the case. The most striking deviation from symmetry is that the brightness of the far surround is constant for the CG experiments- at least for small- and intermediate-sized disks - while for the CD experiments, the Ganzfeld brightness increases slowly.

The results of our brightness-constancy measurements (see Schouten \& Blommaert, 1995) also display an increase of the Ganzfeld brightness with its luminance. These results could be described adequately by means of a model involving an accumulation of contrast information with the addition of a weak luminance-dependent component. To ensure a fair comparison between the results of the $C G$ and $C D$ experiments, this luminance de-

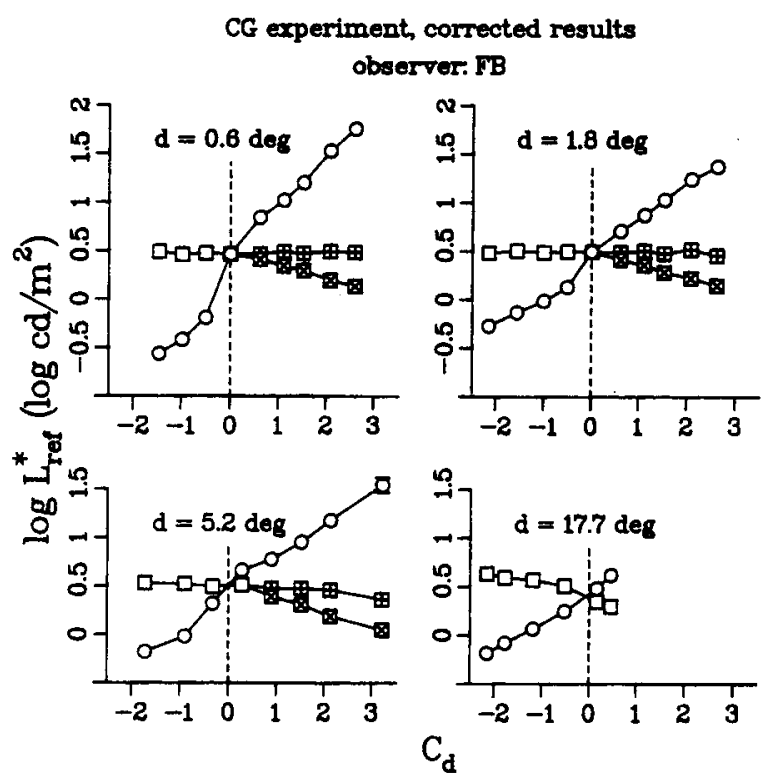

CG experiment, corrected results observer. GS

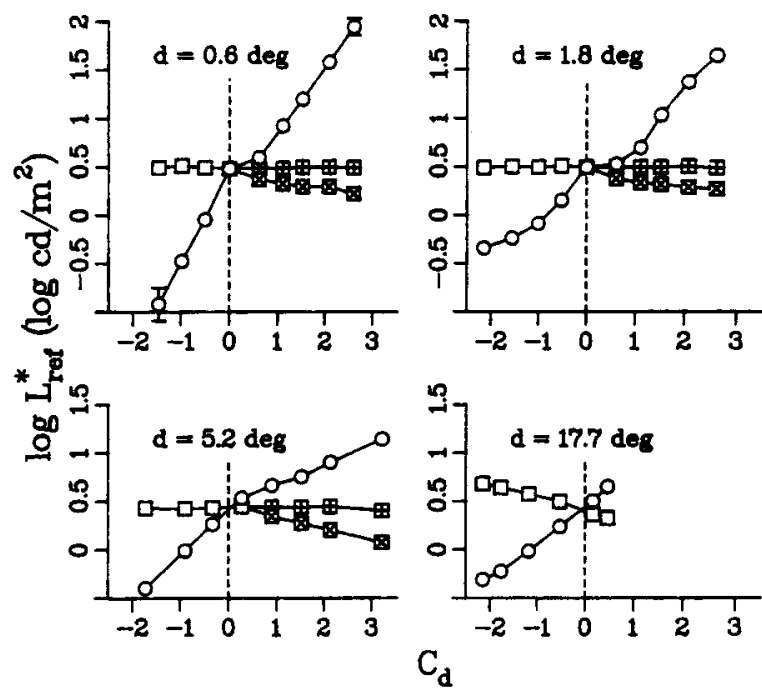

Figure 7. Corrected results of the constant Ganzfeld experiments for Observers F.B. and G.S., with $\log L_{\text {ref }}^{*}$ values plotted as a function of $C_{\mathrm{d}}$ for $d=0.6^{\circ}, 1.8^{\circ}, 5.2^{\circ}$, and $17.7^{\circ}$. Circles are for the disk, while the far surround is marked by a square with an upright cross, and the near surround by a square with a diagonal cross. In the case of a subjectively homogeneous Ganzfeld, the crosses are omitted. 

CD experiment, corrected results
observer: FB

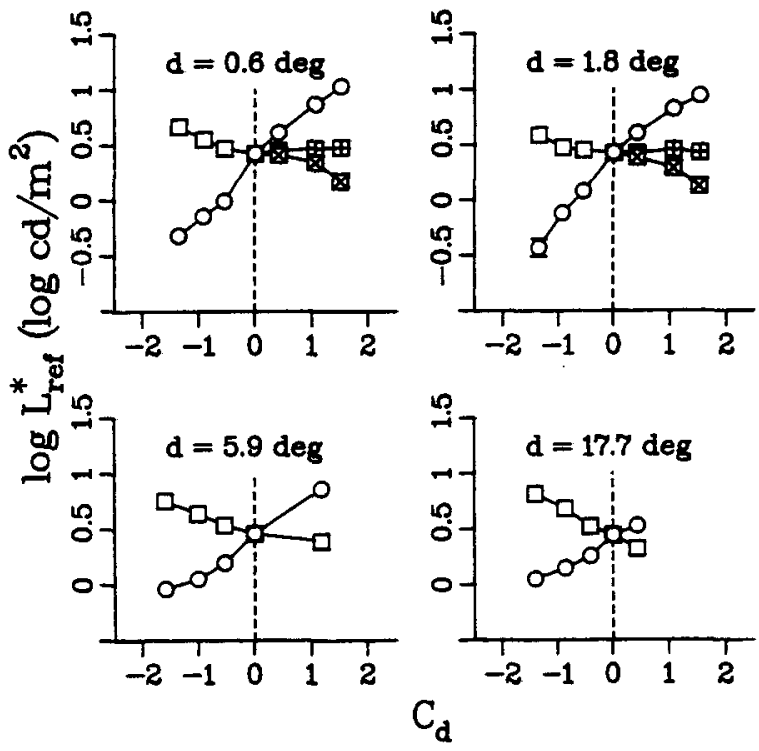

CD experiment, corrected results observer. GS

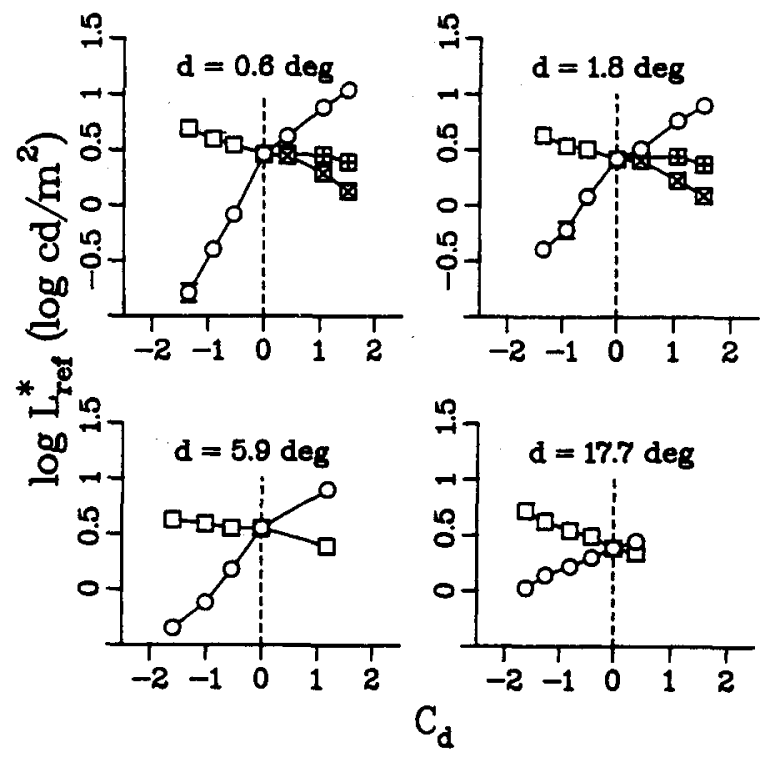

Figure 8. Corrected results of the constant disk experiments for Observers F.B. and G.S., with $\log L_{\text {ref }}^{*}$ values plotted as a function of $C_{\mathrm{d}}$ for $d=0.6^{\circ}, 1.8^{\circ}, 5.9^{\circ}$, and $17.7^{\circ}$. Circles are for the disk, while the far surround is marked by a square with an upright cross, and the near surround by a square with a diagonal cross. In the case of a subjectively homogeneous Ganzfeld, the crosses are omitted.

pendence should be compensated for in the data presented in Figures 4 and 5. Therefore, equivalent $\log L_{\mathrm{ref}}$ values, defined at a fixed level of $L_{\mathrm{G}}=1.0 \mathrm{~cd} / \mathrm{m}^{2}$ and abbreviated to $\log L_{\text {ref }}^{*}$, were calculated. The computation, performed with the simple model presented in our preceding paper on brightness constancy (Schouten
\& Blommaert, 1995), is described in full detail in the Appendix.

Corrected results for the $\mathrm{CG}$ experiments are shown in Figure 7, and those for the $\mathrm{CD}$ experiments are shown in Figure 8 . To stress the similarity between these corrected data, they are plotted as a function of the retinex contrast between the disk and Ganzfeld, defined as $C_{\mathrm{d}}=$ $\log \left(L_{\mathrm{d}} / L_{\mathrm{G}}\right)$. Here, the symbols in the plots represent means of four $\log L_{\mathrm{ref}}^{*}$ values. Standard deviations of these means exceeding the symbol size are indicated by vertical bars. Again, open circles refer to the disk and squares refer to the surround. In particular, squares with an upright cross are for the far surround and squares with a diagonal cross are for the near surround. Although the corrected results of the CG and CD experiments are more alike, there is still some discrepancy. The plots of Figure 8 show, for decreasing, negative values of $C_{\mathrm{d}}$, an increase of the $\log L_{\mathrm{ref}}^{*}$ values concerning the Ganzfeld. On the other hand, $\log L_{\text {ref }}^{*}$ of the far surround is still constant in the graphs of Figure 7, except for diameter $d=17.7^{\circ}$.

To analyze these differences, we plotted the results of all brightness matches of Ganzfelder in a single graph (see Figure 9) as a function of the Ganzfeld luminance
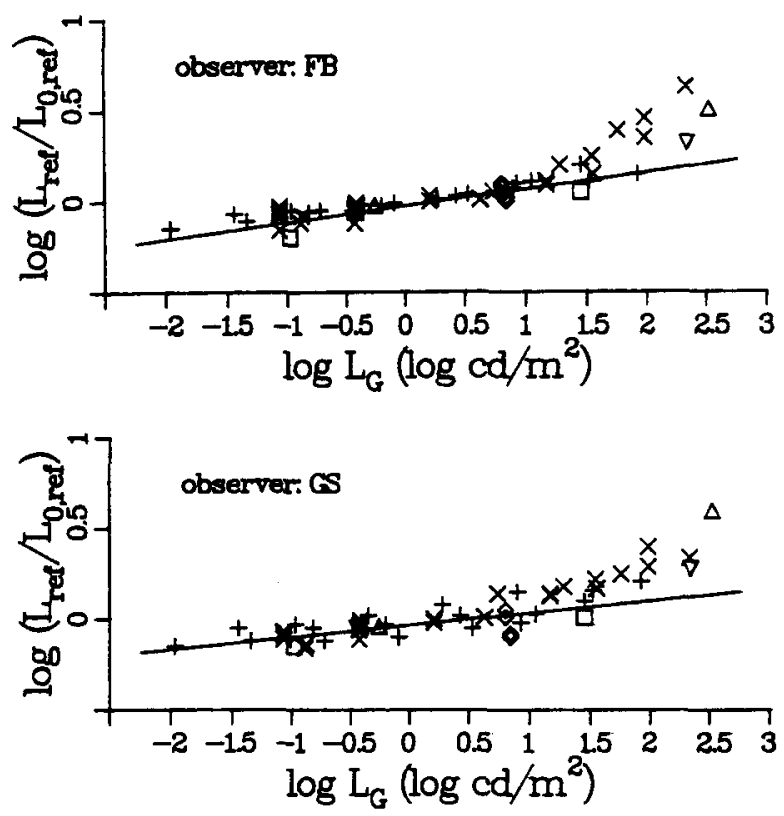

Figure 9. Pooled brightness matches of the Ganzfeld as a function of its luminance for Observers F.B. and G.S. The ordinate represents $\log \left(L_{\text {ref }} / L_{0, \text { ref }}\right)$; that is, the results are corrected for slight variations over the experiments of the luminance $L_{0 \text {,ref }}$. Every symbol represents an average over four matches. Different symbols indicate separate experiments. Upright crosses refer to the brightness-constancy experiment (see Schouten \& Blommaert, 1995). Diagonal crosses are used for the experiments on luminance-range expansion. Since the brightness matches of the Ganzfeld for $d=17.7^{\circ}$ deviate from those concerning smaller disks, they are omitted. Some additional data points are also presented (see Schouten, 1993). Furthermore, the model prediction, based solely on the brightness-constancy measurements, is indicated by the solid line. 

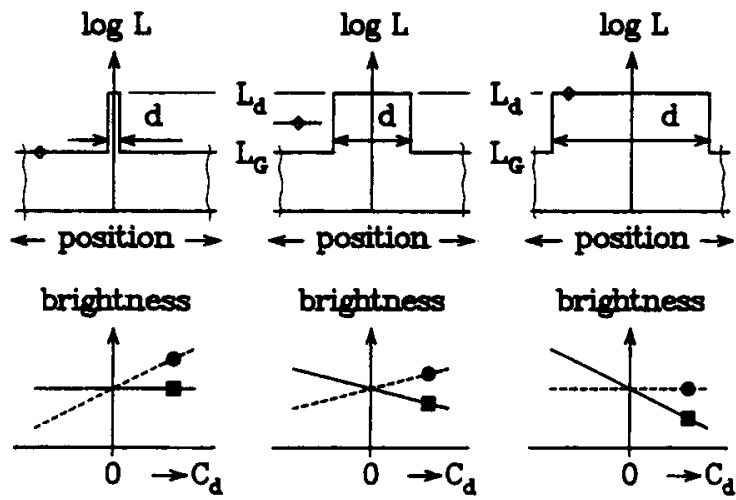

Figure 10. Schematic brightness changes of disk and surround for variations of the diameter $d$. In order to present a clear picture, the brightness-indention effect is not included and only properly corrected brightness values are considered. Luminance profiles for increasing $d$ values are drawn in the upper part. The luminance level, which should be rescaled to a fixed value, is indicated by a diamond. The size of the visual field is marked by wavy lines. Below, analogous to Figures 7 and 8 , brightness values are plotted against $C_{\mathrm{d}}$. A solid line is used for the surround brightness and a dashed line for the brightness of the disk. The symbols - a circle for the disk and a square for the surround-specifically refer to the above luminance profiles.

$L_{\mathrm{G}}$. Different symbols refer to separate experiments; details are given in the figure caption. This collection of brightness matches shows that the model, on which the correction is based, and which generates the straight line in the plots, is inaccurate for large values of $L_{\mathrm{G}}$. It should be noted that the deflecting points in Figure 8, derived by means of this first-order description (see the Appendix), actually fall within the deviating high-luminance region. In the light of these findings, we feel justified in concluding that the results of the two types of experiments can be considered internally consistent.

\section{Anchorage Onto the Brightness Scale}

Schouten and Blommaert (1995) have already shown that, for small disks on a Ganzfeld, the Ganzfeld brightness is, to a first-order approximation, anchored onto the brightness scale. In other words, the brightness of the Ganzfeld is independent of the contrast of a small superimposed disk. This is illustrated in the left-hand panel of Figure 10: The Ganzfeld brightness of the configuration (top) produces a horizontal line in the lower graph. The corrected results for small disks shown in Figure 7 (where the aforementioned deviations at high luminance levels can be neglected) are in accordance with this schematic graph. On the other hand, if the diameter $d$ approaches the width of the entire visual field, as demonstrated in the right-hand panel of Figure 10, it is the brightness of the disk, stretched out almost to the size of a Ganzfeld, that should remain constant (if corrections are carried out for variations of the luminance $L_{\mathrm{d}}$ ). Thus, in this case, the luminance $L_{\mathrm{d}}$ is transformed to the anchorage on the brightness scale. The brightness of the surround, shrunk to a thin peripheral annulus, now varies with the contrast $C_{\mathrm{d}}$. These two extreme states change into one another as the diameter $d$ gradually increases. This implies that, for the spatial configuration pictured in the middle panel of Figure 10, the brightness of the disk as well as its surround vary as a function of $C_{\mathrm{d}}$, and that some intermediate luminance level $\bar{L}$ is projected onto the anchored brightness level. This behavior corresponds to the trends that can be seen in Figures 7 and 8 for $d=17.7^{\circ}$. Since this effect is only just emerging, measurements with larger disks are necessary in order to derive a useful empirical expression for the average luminance level $\bar{L}$.

\section{Size Effects on Brightness Contrast}

Here, it is assumed that brightness contrast or perceived contrast corresponds to the brightness difference between the disk and the near surround. A reliable measure for this brightness difference is $\Delta \log L_{\text {ref }}^{*}$ (i.e., the difference between the $\log L_{\text {ref }}^{*}$ value of the disk and the $\log L_{\text {ref }}^{*}$ value of the nearby surround, as depicted in Figures 7 and 8 ). In Figure $11, \Delta \log L_{\text {ref }}^{*}$ is plotted against the retinex contrast $C_{\mathrm{d}}=\log \left(L_{\mathrm{d}} / L_{\mathrm{G}}\right)$, and the diameter $d$ of the disk is used as a parameter. Only averaged val-
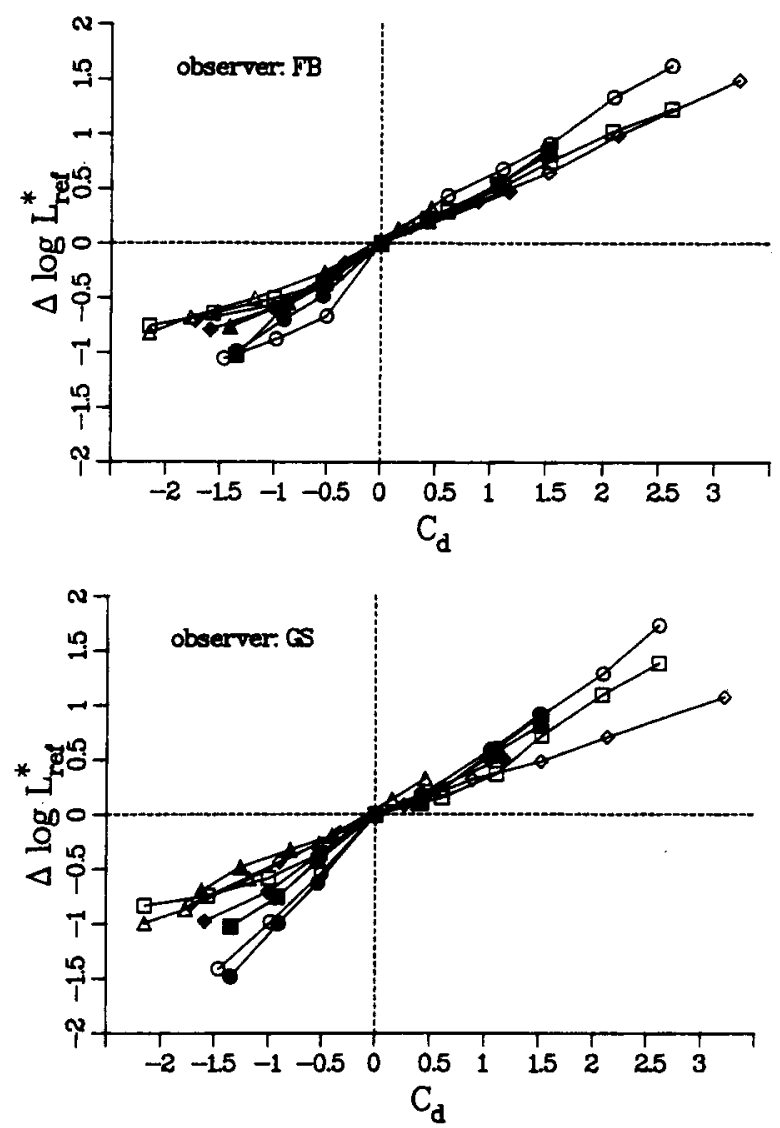

Figure 11. $\Delta \log L_{\text {ref }}^{*}$ versus $C_{\mathrm{d}}$ for Observers F.B. and G.S. Averaged values of four corresponding differences are plotted. Open symbols refer to the constant Ganzfeld experiments and filled symbols to the constant disk experiments. Circles represent a diameter of $0.6^{\circ}$, squares a diameter of $1.8^{\circ}$, open diamonds a diameter of $5.2^{\circ}$, filled diamonds a diameter of $5.9^{\circ}$, and triangles a diameter of $17.7^{\circ}$. 
ues of four corresponding differences are marked. Open symbols refer to the $\mathrm{CG}$ experiments, filled symbols to the CD experiments. Circles indicate a diameter of $0.6^{\circ}$, squares a diameter of $1.8^{\circ}$, open diamonds a diameter of $5.2^{\circ}$, filled diamonds a diameter of $5.9^{\circ}$, and triangles a diameter of $17.7^{\circ}$. Note that the plotted differences roughly concur for the $C G$ and $C D$ experiments. For small values of $d$, brightness contrast is somewhat greater.

Many studies on brightness using disks with variable diameters were dedicated to the spatial Broca-Sulzer effect (Björklund \& Magnussen, 1979; du Buf, 1987; Hanes, 1951; Higgins \& Rinalducci, 1975). For this smallscale size effect, diameters varied from 1 to approximately $30 \mathrm{~min}$ of arc. Changes in spatial characteristics at a larger scale, as employed in our experiments, usually aim at size invariance. For perceived grating contrast, it was shown that size invariance applies, at least for spatial frequencies down to about $1 \mathrm{c} / \mathrm{deg}$ (Georgeson \& Sullivan, 1975; Kulikowski, 1976; Quinn, 1985). On the other hand, minor effects of diameter are found here for disks on a Ganzfeld. However, compared with the above suprathreshold studies, the range of stimulus sizes applied in our experiments was much larger.

\section{CONCLUSION}

This investigation reveals that, besides brightness constancy, there is an additional compression mechanism in the luminance-brightness mapping. We have termed this new compression mechanism brightness indention. It occurs if the Ganzfeld is less luminous than the disk and causes a brightness lowering of the nearby surround of the disk. No such effect is found when the Ganzfeld is brighter than the disk. As a side issue of this investigation, it is shown that (1) for smalland intermediate-sized disks, the Ganzfeld brightness is anchored onto the brightness scale, whereas for the largest disk (about $20^{\circ}$ ), slight deviations occur; and (2) there is a small effect of diameter on brightness contrast.

\section{REFERENCES}

Bartleson, C. J., \& Breneman, E. J. (1967). Brightness perception in complex fields. Journal of the Optical Society of America, 57, 953957.

Björklund, R. A., \& Magnussen, S. (1979). Decrement versions of the Broca-Sulzer effect and its spatial analogue. Vision Research, $19,155-157$.

DU BuF, J. M. H. (1987). Spatial characteristics of brightness and apparent-contrast perception. Unpublished doctoral dissertation, Eindhoven University of Technology, Eindhoven, The Netherlands.

FeCHNER, G. T. (1860). Elemente der Psychophysik. Leipzig, Germany: Breitkopf \& Hartel.

FIoRENTINI, A. (1972). Mach band phenomena. In D. Jameson \& L. M Hurvich (Eds.), Handbook of sensory physiology (Vol. 7, pp. 188201). Berlin: Springer-Verlag.

Forsyth, D. A. (1990). Colour constancy. In A. Blake \& T. Troscianko (Eds.), Al and the eye (pp. 201-229). Chichester, U.K.: Wiley.

Georgeson, M. A., \& Sullivan, G. D. (1975). Contrast constancy: Deblurring in human vision by spatial frequency channels. Journal of Physiology, 252, 627-656.
HANES, R. M. (1951). Suprathreshold area brightness relationships. Journal of the Optical Society of America, 41, 28-31.

HeinemanN, E. G. (1972). Simultaneous brightness induction. In D. Jameson \& L. M. Hurvich (Eds.), Handbook of sensory physiol$o g y$ (Vol. 7, pp.146-169). Berlin: Springer-Verlag.

Higgins, K. E., \& Rinalducci, E. J. (1975). Suprathreshold intensityarea relationships: A spatial Broca-Sulzer effect. Vision Research, 15, 129-143.

Horeman, H. W. (1965). Relations between brightness and luminance under induction. Vision Research, 5, 331-340.

Jameson, D., \& Hurvich, L. M. (1961). Complexities of perceived brightness. Science, 133, 174-179.

Krueger, L. E. (1989). Reconciling Fechner and Stevens: Toward a unified psychophysical law. Behavioral \& Brain Sciences, 12, 251. 320.

Kulikowski, J. J. (1976). Effective contrast constancy and linearity of contrast sensation. Vision Research, 16, 1419-1431.

LowRY, E. M., \& DePALMA, J. J. (1961). Sine-wave response of the visual system: I. The Mach band phenomenon. Journal of the Optical Society of America, 51, 740-746.

MCCANN, J. J. (1988). Calculated color sensations applied to image reproduction. Proceedings of the SPIE: Image Processing Analysis, Measurement, and Quality, 901, 205-214.

QuinN, P. C. (1985). Suprathreshold contrast perception as a function of spatial frequency. Perception \& Psychophysics, 38, 408-414.

RatLiFf, F. (1984). Why Mach bands are not seen at the edges of a step. Vision Research, 24, 163-165.

RouFs, J. A. J., BlommaERT, F. J. J., \& DE RidDER, H. (1991). Brightnessluminance relations: Future developments in the light of the past. CIE Proceedings (22nd Session, Melbourne), 2, 135-148.

SAUNDERS, J. E. (1972). The validity of magnitude estimations of luminosity and the measurement of the relative effects of preadaptation and contrast. Vision Research, 12, 689-698.

Schouten, G. (1993). Luminance-brightness mapping: The missing decades. Unpublished doctoral dissertation, Eindhoven University of Technology, Eindhoven, The Netherlands.

Schouten, G., \& Blommaert, F. J. J. (1995). Brightness constancy in a Ganzfeld environment. Perception \& Psychophysics, 57, 1112-1122.

Stevens, S. S. (1957). On the psychophysical law. Psychological Review, 64, 153-181.

WAGENAAR, W. A. (1975). Stevens vs. Fechner: A plea for dismissal of the case. Acta Psychologica, 39, 225-235.

Walraven, J., Enroth-Cugell, C., Hoon, D. C., Macleod, D. I. A., \& SchNAPF, J. L. (1990). The control of visual sensitivity: Receptoral and postreceptoral processes. In L. Spillman \& J. S. Werner (Eds.), The neurophysiological foundations of visual perception (pp. 53-100). London: Academic Press.

\section{NOTES}

1. For the largest disk, the distance $l$ of the eye to the circular contour (i.e., the edge of the hole in the hemisphere) was about $3.5 \mathrm{~cm}$. Application of locking caps yielded an enlargement of the distance $l$ to $8.4 \mathrm{~cm}$. In the case of the tiny disks, $l$ refers to the distance between the eye and the monitor, which was approximately $1.20 \mathrm{~m}$. The distances $l=3.5$ and $8.4 \mathrm{~cm}$ are both beyond the accommodation range of the normal young adult's eye $( \pm 20 \mathrm{~cm}$ to $\infty)$. Therefore, in these situations, the optics of the eye cause a considerable amount of blur on the retina, especially for $l=3.5 \mathrm{~cm}$.

2. However, for disk-like stimuli, the influence of edge-sharpness on brightness is of minor importance (du Buf, 1987).

3. A strong, convincing Mach-band effect is perceived at the two ends of a "ramp" in luminance from one uniform level to another.

\section{APPENDIX \\ Calculation of $\log L_{\text {ref }}^{*}$ Values}

In the model presented by Schouten and Blommaert (1995), brightness is constructed by means of an accumulation of contrast information with a weak luminance-dependent factor. It was shown that application of this concept to the experimental 
situation (i.e., brightness matches of the familiar reference stimulus with a test stimulus that consisted of a Ganzfeld with small disks superimposed) can be summarized with the following expression:

$$
C_{\mathrm{ref}}=\frac{\alpha\left(L_{\mathrm{G}}\right)}{\alpha_{\mathrm{ref}}}\left(C_{\mathrm{G}}+C_{\mathrm{d}}\right)-K
$$

where $C_{\text {ref }}$ denotes the retinex contrast between the reference disk and the $9^{\circ}$ background field-that is,

$$
C_{\text {ref }}=\log \frac{L_{\text {ref }}}{L_{0, \text { ref }}} .
$$

Likewise, $C_{d}$ is the retinex contrast in the test stimulus between the considered disk and the homogeneous surround-that is,

$$
C_{\mathrm{d}}=\log \frac{L_{\mathrm{d}}}{L_{\mathrm{G}}} .
$$

Furthermore, $\alpha_{\text {ref }}, C_{\mathrm{G}}$, and $K$ are regarded as constants, and the factor $\alpha\left(L_{\mathrm{G}}\right)$, which depends on the Ganzfeld luminance $L_{\mathrm{G}}$ of the test stimulus, is given by

$$
\alpha\left(L_{\mathrm{G}}\right)=\hat{\alpha}\left(\log L_{\mathrm{G}}-\log L_{\mathrm{th}}\right),
$$

with $\hat{\alpha}$ and $L_{\text {th }}$ constant. Equations 1 and 4 imply that the difference $\Delta C_{\text {ref }}$, between the $C_{\text {ref }}$ value of the disk and the Ganzfeld (for which $C_{\mathrm{d}}=0$ ), increases linearly with $\log L_{\mathrm{G}}$ namely:

$$
\Delta C_{\mathrm{ref}}=\frac{\hat{\alpha}}{\alpha_{\mathrm{ref}}}\left(\log L_{\mathrm{G}}-\log L_{\mathrm{th}}\right) C_{\mathrm{d}} .
$$

The scale factor $p$ that transforms a difference $\Delta C_{\text {ref }}^{(1)}$ at a Ganzfeld luminance $L_{\mathrm{G}}^{(1)}$ to a corresponding difference $\Delta C_{\mathrm{ref}}^{(2)}$ at a value $L_{\mathrm{G}}^{(2)}$ is given by
Table A1

Parameter Values for Observers F.B. and G.S.

\begin{tabular}{ccc} 
Observer & $\log L_{\text {th }}$ & $K$ \\
\hline F.B. & -5.07 & 0.49 \\
G.S. & -21.2 & 0.90 \\
\hline
\end{tabular}

Note--See also Table 1, second and fourth column, in Schouten and Blommaert, 1995.

$$
p=\frac{\log L_{\mathrm{G}}^{(2)}-\log L_{\mathrm{th}}}{\log L_{\mathrm{G}}^{(1)}-\log L_{\mathrm{th}}} .
$$

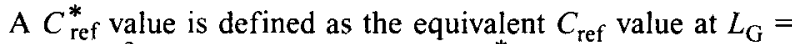
$1.0 \mathrm{~cd} / \mathrm{m}^{2}$. In order to obtain these $C_{\text {ref }}^{*}$ values, we need, besides $p$ factors with $\log L_{\mathrm{G}}^{(2)}=0$, an anchorage with respect to which the scaling must be performed. Note that for $C_{\mathrm{d}}=-C_{\mathrm{G}}$, Equation 1 reduces to $C_{\text {ref }}=-K$; that is, $C_{\text {ref }}$ is a constant, independent of the Ganzfeld luminance $L_{\mathrm{G}}$. Therefore, $C_{\text {ref }}^{*}$ values are generated by

$$
C_{\text {ref }}^{*}=p\left(C_{\text {ref }}+K\right)-K,
$$

with

$$
p=\frac{\log L_{\mathrm{th}}}{\log L_{\mathrm{th}}-\log L_{\mathrm{G}}} .
$$

Here, $L_{\mathrm{G}}$ refers to the Ganzfeld luminance; the constants $\log L_{\text {th }}$ and $K$ are listed in Table AI for both the observers (F.B. and G.S.). Finally, equivalent luminances of the reference disk on a logarithmic scale (i.e., $\log L_{\text {ref }}^{*}$ values) are obtained by performing the inverse operation of Equation 2-that is,

$$
\log L_{\text {ref }}^{*}=C_{\text {ref }}^{*}+\log L_{0, \text { ref }} .
$$

